\title{
Rethinking Cancer Immunotherapy by Embracing and Engineering Complexity
}

\author{
Matthew H. W. Chin ${ }^{1,2^{*}}$, Eileen Gentleman ${ }^{3}$, Marc-Olivier Coppens ${ }^{2,4}$, Richard M. Day ${ }^{1,2}$ \\ ${ }^{1}$ Centre for Precision Healthcare, Division of Medicine, University College London, London, United \\ Kingdom \\ ${ }^{2}$ Centre for Nature Inspired Engineering, University College London, London, United Kingdom \\ ${ }^{3}$ Centre for Craniofacial and Regenerative Biology, King's College London, London, United \\ Kingdom \\ ${ }^{4}$ Department of Chemical Engineering, University College London, London, United Kingdom
}

Keywords: Bioengineering, Complex Systems, Holism, Immunotherapy, Process Intensification

*Correspondence: matthew.chin.15@ucl.ac.uk (M. H. W. Chin)

ORCID: 0000-0003-1633-7786 (M. H. W. Chin), 0000-0003-0447-5137 (E. Gentleman), 0000-

0002-1810-2537 (M.-O. Coppens), R. M. Day (0000-0002-3124-2294)

Twitter: @MatthewHWChin (M. H. W. Chin), @GentlemanLab (E. Gentleman), @UCLCNIE (M.-

O. Coppens)

Lab websites: http://cnie.org.uk (M.-O. Coppens), http://ucl.ac.uk/day-lab (R. M. Day)

\begin{abstract}
The meteoric rise of cancer immunotherapy in the last decade has led to promising treatments for a number of hard-to-treat malignancies. In particular, adoptive $T$ cell therapy has recently reached a major milestone with two products approved by the FDA. However, the inherent complexity of cell-based immunotherapies means that their manufacturing time, cost, and controllability limit their effectiveness and geographic reach. One way to address these issues may lie in complementing the dominant, reductionistic mentality in modern medicine with complex systems thinking. In this Opinion, we identify key concepts from complexity theory to address manufacturing challenges in cell-based immunotherapies and raise the possibility of a unifying framework upon which future bioprocessing strategies may be designed.
\end{abstract}




\section{Highlights}

Complexity theory provides a conceptual framework in which biological and artificial networks may be designed or manipulated to intensify cell bioprocessing in cancer immunotherapies.

Studies on T cell mechanobiology have revealed how physical parameters may be exploited to perturb intracellular networks as an effective way of controlling $T$ cell fates for immunotherapeutic applications.

Systems biology-based computational models open up the potential to predict cues needed to guide $T$ cell differentiation and reprogramming.

Advances in immunomodulatory biomaterials, microfabrication and wearable medical technologies raise the possibility of scaling up point-of-care deployment of immunotherapies. 


\section{Complexity and its relevance to cancer immunotherapy}

Immunotherapy is widely regarded as one of the most important breakthroughs in cancer therapy, evidenced by encouraging clinical results for checkpoint inhibitors [1] and T cell-based adoptive cell transfer (ACT) [2]. ACT involves direct use of immune cells to eradicate cancer cells. Three types of ACT are currently being developed for immunotherapy - tumour infiltrating lymphocytes (TILs), T cell receptor (TCR)-transduced T cells and chimeric antigen receptor (CAR) T cells. TIL therapy enriches naturally occurring tumour-reactive $T$ cells extracted from tumours, whereas CAR- and TCR-transduced T cells are gene modified T cells equipped with custom tumour antigen-targeting receptors. Promising clinical data with CAR-T ACT [3] has led to US Food and Drug Administration (FDA) approval for two products, Kymriah (Novartis) (https://bit.ly/2W4J2Dz) and Yescarta (Gilead) (https://bit.ly/2W4J2Dz).

The manufacture of ACT drugs pose unique challenges in scalability, accessibility and biological control. Manufacturing typically involves ex vivo stimulation to activate expansion and differentiation of patient-derived $T$ cells before reinfusion into the patient. The components of the therapy (cells, patients, logistics, manufacturing) are all systems with interdependent parts - complex systems.

This Opinion article aims to promote lateral thinking around the challenges of ACT manufacture by framing them within a unified conceptual framework - Complexity Theory. We draw insight from systems biology and mechanobiology to highlight the importance of biomolecular networks and microenvironments in producing potent therapeutic T cells. From there, we propose the use of artificial cell niches as a systems-based approach to enhance T cell persistence. Moreover, the locus of ACT manufacturing may be shifted from linear, centralised production to distributed, on-demand networks, similar to the organisation of biological networks. Enabling such vision, we describe how manufacturing may be performed in a point-of-care and agile fashion by extending the immune system with wearable microfluidic "factories". 

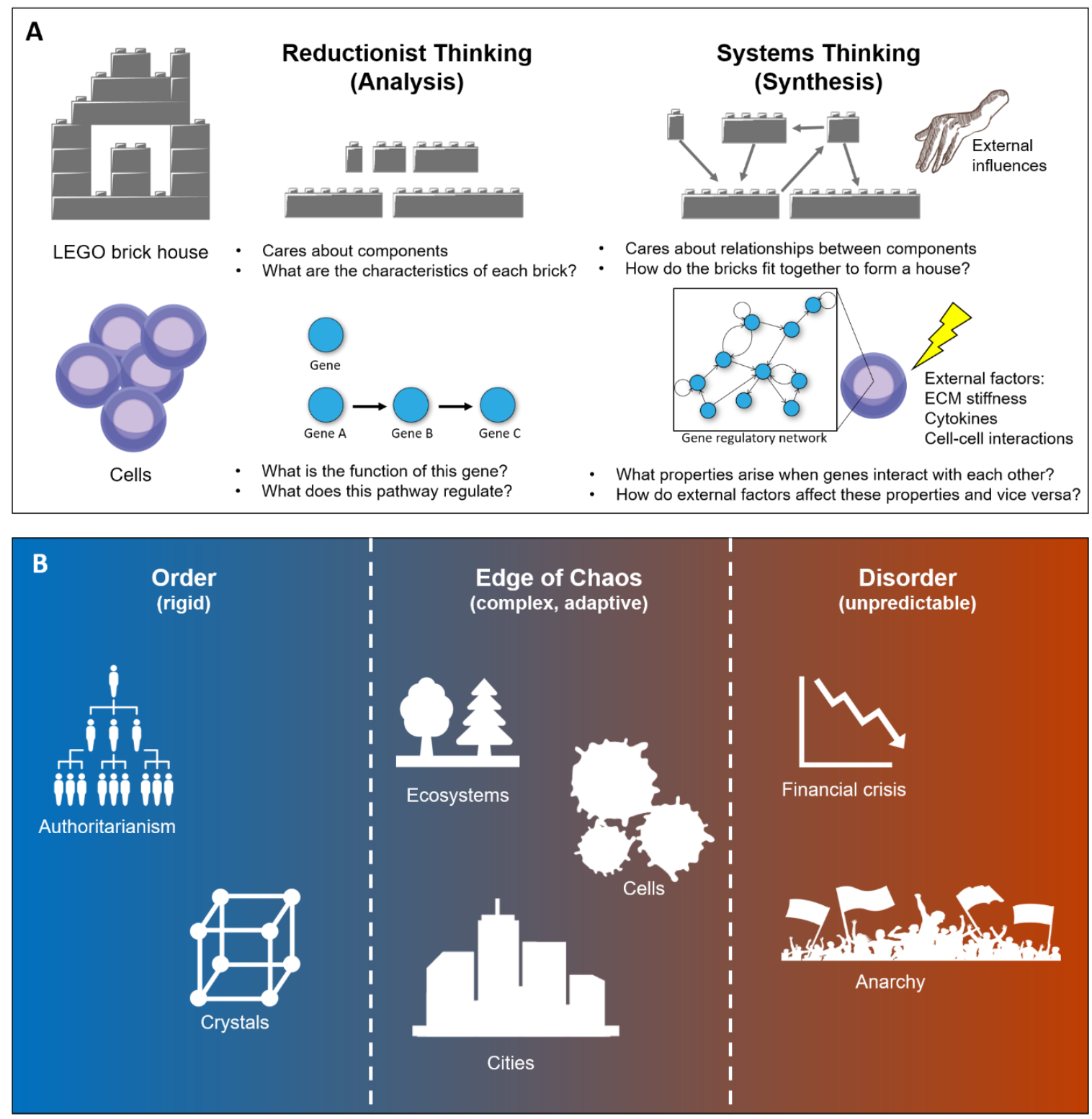

Figure $1 \mathrm{~A}$ ) Differences between reductionist thinking and systems thinking. LEGO ${ }^{\circledR}$ brick house: Although studying individual bricks of simple systems might already be sufficient to predict some possible configurations or properties of the assembled structure, there is no information about the concept of a "house" contained in individual bricks. It is the relations between the bricks that generate the house. Cells: Cells contain a large number of mutually interacting components, including genes, which give rise to emergent properties. To understand these higher-order properties requires an understanding of relations between components. B) The edge of chaos is the transition zone between order and disorder. This interface between the two regimes is hypothesised to be where complexity is maximised. Here, systems can evolve and adapt within limits via a dynamic interplay between order and disorder. Too much order could lead to stagnation, due to the high number of constraints imposed on the system. However, too little order could lead to disastrous consequences. Some systems, even deterministic ones, might be particularly sensitive, where a small change in the initial conditions can lead to a dramatic, unpredictable response (chaos) in a later state. This phenomenon has been likened to an imaginary scenario where a tornado or hurricane is generated in Texas due to a butterfly flapping its wings in Brazil, hence the name "butterfly effect". On the other hand, chaotic systems can also be controlled by exerting only small external influences (triggers) to target and stabilise widely different outcomes, which is a great opportunity (chaos control). 
Complexity has broad relevance, in fields as diverse as physics [4], humanities [5], economics [6] and biology [7]. Despite superficial differences, many artificial (e.g. supply chains, the World Wide Web) and biological systems (e.g. gene regulatory networks (GRNs), the immune system) share deep commonalities typical of complex systems, such as nonlinearity, co-evolution and emergence [8]. Nevertheless, the prevailing structure of scientific enquiry, rooted in reductionism (Error! Reference source not found.), focuses on parts and fails to capture emergent properties that arise from system interdependencies - this is particularly true for medicine $[9,10]$. For instance, genomic, proteomic and graph theoretical analyses suggest that regulatory molecular networks in the cell typically form a huge "connected component" that spans the majority of the genome and coordinates cell fate in a global manner [11,12]. Yet, "precision" therapeutics have been developed based on single molecular targets, and the notion that there is a linear causal link between genotype and phenotype. The case for a systems perspective is reinforced by the fact that target-selective drugs have so far demonstrated limited efficacy against cancer [13-15].

The use of self-organising complex systems (immune cells) in ACT to combat cancer is a step towards systems thinking for cancer therapy. However, ACT research predominantly remains reductionist at the molecular and cellular levels. This is reflected by the focus on developing CAR constructs for precise tumour targeting [2] as well as large-scale expansion of CD8 ${ }^{+}$cytotoxic $T$ cells for tumour destruction [16]. However, the phenotype of T cells remains important [17]. In particular, there is considerable interest in generating early-lineage $T$ cells with a stem-cell memory or central memory phenotype, as there is growing evidence suggesting the $T$ cell differentiation is inversely related to proliferative potential and anti-tumour toxicity $[18,19]$. It is currently difficult to systematically predict and consistently generate phenotypic attributes that achieve targeted clinical responses [20]. A major reason for limited phenotypic control is that the complex, multiscale signalling networks governing $T$ cell behaviour are not fully accounted for under the current reductionistic paradigm [21].

\section{Cell fate determination: a dynamical systems perspective}


Optimisation of T cell phenotype for ACT requires an holistic approach. Its applicability to biology was proposed by Conrad Waddington more than 80 years ago: "to say that an animal is an organism means in fact two things: firstly, that it is a system made up of separate parts, and secondly, that in order to describe fully how any one part works one has to refer either to the whole system or to the other parts" [22]. Here, Waddington was describing precisely the irreducible characteristics of complex systems. This thinking culminated in his conception of the "epigenetic landscape" - a metaphor to illustrate the phenotypic change of an embryonic stem cell during development (Figure 2A and B). Three crucial ideas are presented by the metaphor - (i) the landscape is an abstraction of a higher-order property of the underlying nonlinear gene interactions, (ii) cell types are discrete stable states, and (iii) a slope/threshold must be overcome for a cell to go from one valley to another. This last point essentially relates to cell reprogramming.

Waddington's landscape is more than just a metaphor - it has a mathematical basis and is supported by cell differentiation experiments (Box 1). Using the formalism of state space, Kauffman and Huang have constructed models based on the "attractor landscape", or "potential landscape", to describe GRN dynamics underlying phenotypic transitions (Figure 2C). The attractor landscape model suggests that cell fate regulation is, in principle, multi-directional and reversible under the right conditions [11,23]. A change in cell fate is essentially a transition from one attractor to another over time. A cell can switch phenotype through a change in its network state (gene expression profile) without any modification to the landscape topology (GRN wiring) [24]. Alternatively, a cell may be primed for a different attractor when the epigenetic landscape changes due to mutation (network re-wiring) or a change in gene-gene correlation (network connection strength), hence destabilising the current attractor [25,26]. Moreover, Furusawa and Kuneka have summarised possible mechanisms through which stem cells unite dynamic robustness (self-renewal capacity) with flexibility (differentiation capacity) in the attractor model [27]. Thus, in the dynamical systems framework, it is unsurprising that cell types can be reprogrammed (e.g. induced pluripotent stem cells, iPSCs). Putting network biology into practice, there already exist computational algorithms (e.g. Mogrify) that use gene expression data and regulatory network information to predict the optimal 
combinations of transcription factors for direct cell reprogramming (transdifferentiation).

The dynamical systems perspective of cell reprogramming has major implications for phenotypic control in T cell-based ACT. In the future, T cell manufacture may be guided by computational models that predict the combination of differentiation cues needed to achieve a potent anti-tumour response. Furthermore, this could lessen the burden on logistics by offering alternative cell sources when limited quantities are available from patients affected by disease or chemotherapy [30]. 
A

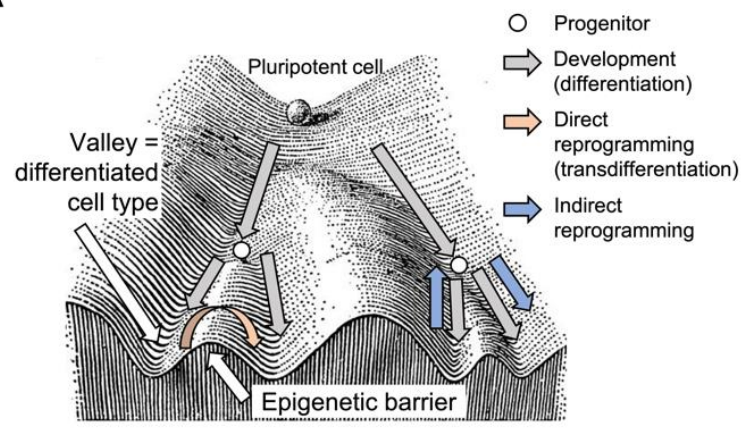

B

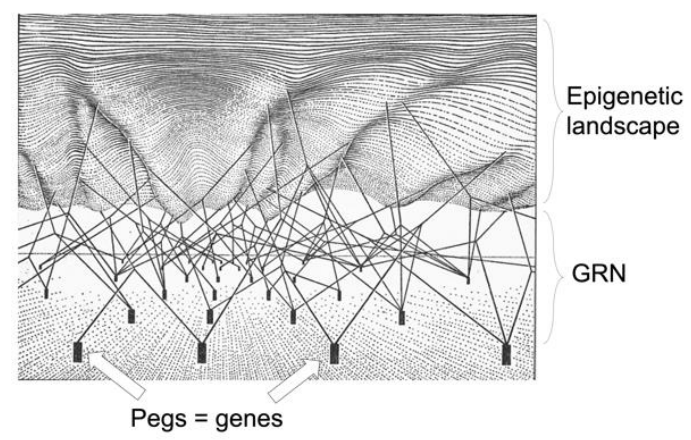

C GRN topology High (N)-dimensional state space

Quasi-potential landscape

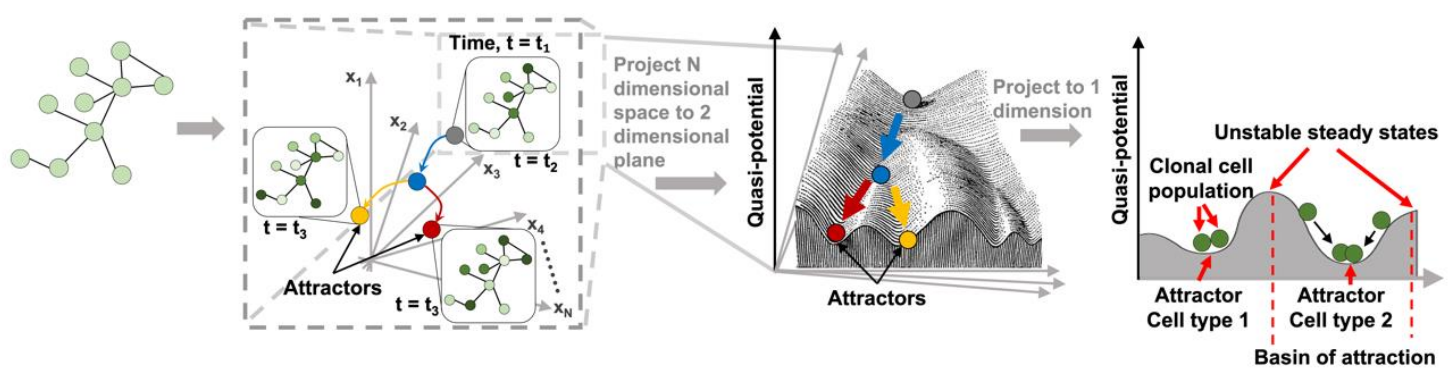

Figure 2 A) Waddington's epigenetic landscape. The landscape itself and the marble at the top are from his original diagram in "The Strategy of the Genes". In the landscape, a marble (pluripotent cell) rolls down the gradient, where the elevation is inversely proportional to cell maturity. Following a specific developmental trajectory (chreod; grey arrow) down the landscape, the marble encounters branching points where it adopts a multipotent state, before settling down in one of the valleys (cell types). Cell types are separated by hills (epigenetic barrier) that restrict direct reprogramming (orange arrow) of cell types. Indirect reprogramming (blue arrow) is achieved when a differentiated cell converts into a progenitor cell before differentiating into the target cell type. B) The topology of the landscape depends on the constrained interactions of the underlying GRN. C) For a GRN consisting of $\mathrm{N}$ genes, the regulatory interactions between the genes can be drawn as a graph of nodes and edges to form the network topology. The interactions can be activating or inhibitory in nature and, therefore, different gene expression profiles (network states) can arise as the GRN moves through time. At various time points, the state of the network (grey, blue, yellow and red balls) can be mapped into a state space of $\mathrm{N}$ dimensions, with dimension $\mathrm{i}$ (coordinate $\mathrm{x}_{\mathrm{i}}$ ) representing the expression level of gene $i$ in the network. To depict network state, the brightness of different green nodes is changed at each time point to indicate a change in gene expression profile. When the pluripotent cell (grey; the initial state) becomes a progenitor cell (blue), it is in a metastable state that is poised for transition into one of the two closest attractors (yellow or red). For visualisation purposes and simplicity, the $\mathrm{N}$ dimensions are projected to a two-dimensional plane and a one-dimensional profile. The quasi-potential for each network state can then be computed and represented as the elevation of the epigenetic landscape. Each attractor is surrounded by a corresponding basin of attraction. Cells in the basin will eventually end up and remain at the bottom of the valley, unless their regulatory network receives a large enough perturbation to escape, or when rewiring of the GRN causes the valley to "flatten".

\section{Controlling T cell fate with engineered extracellular matrix cues}


Although systematic and predictive control over cell type is attractive for ACT, current protocols for reprogramming often rely on gene delivery methods (e.g. viral transduction) and/or cocktails of soluble factors that increase manufacturing cost and time. Elsewhere, physical cues are increasingly exploited to guide, and improve the efficiency of, cell reprogramming of adult stem cells and iPSCs [28]. It might, therefore, be beneficial for ACT to venture beyond the molecule-centric paradigm and employ extracellular physical signals during bioprocessing. Here, we discuss the potential of synthetic cell niches to provide combined biochemical and mechanical cues for ACT manufacture.

In bioprocessing, materials-based strategies can be used to deliver the stimulatory queues required for $\mathrm{T}$ cell differentiation. Indeed, current manufacturing protocols regularly implement ancillary materials to deliver stimulatory signals, often using microbeads coated with monoclonal antibodies against CD3 and CD28 $[29,30]$. However, the design of these materials has overlooked the relevance of physical factors (e.g. substrate stiffness, topography and spatial ligand organisation) in regulating $T$ cell activation. Instead, manufacturing strategies have largely focused on controlling the cytokine environment to direct T cell differentiation (e.g. IL-15 for memory $C D 8^{+} T$ cells [31]).

Although the response of adherent cells to mechanical cues has been extensively studied (Box 2), T cell mechanobiology is an emerging field, and the molecular mechanisms underlying mechanotransduction remain to be elucidated. Nevertheless, accumulating evidence suggests that forces are central to $T$ cell activation and that the TCR-CD3 complex acts as a mechanosensor [32]. Furthermore, cultures on soft materials (e.g. silicone elastomer, hydrogel) presenting stimulatory cues (anti-CD3/CD28) have revealed that matrix stiffness influences key signalling events downstream of TCR-CD3 during activation $[33,34]$. Linking mechanics to T cell differentiation, Harrison and colleagues have discussed how forces generated by cytoskeletal rearrangements following TCR engagement can be propagated to various organelles (e.g. the nucleus, endoplasmic reticulum, mitochondria) to modulate $T$ cell function and gene expression [35]. Indeed, lamin A and the linker of nucleoskeleton and cytoskeleton 
(LINC), which mediate nuclear-cytoskeletal mechanical interactions, have been implicated in the regulation of T cell activation [36].

To date, stiffness-tunable hydrogels that exploit $\mathrm{T}$ cell mechanobiology have only been investigated in non-clinical studies. Applying the systems concept, it is conceivable that future computational tools may extend the Mogrify-like network biology approach to predict optimal combinations of physical and biochemical properties of materials needed to optimise control of $\mathrm{T}$ cell fate. This approach could help address existing challenges of maintaining/extending in vivo persistence of adoptive transferred T cells and complement existing efforts, based on gene transfer or cytokines [37]. The potential of a material-assisted approach has been made more compelling by recent findings that soft biomaterials can rescue exhausted T cells from patients with chronic lymphocytic leukaemia [38]. Computation of the precise combination of cues (e.g. matrix stiffness, ligand density) is needed to tip the cell from one attractor state into the basin of attraction of a desired attractor (e.g. memory T cell). Efforts elsewhere have started to predict mesenchymal stem cell fate in response to mechanical stimuli and culture durations [39].

\section{Systems thinking to address ACT supply and manufacturing challenges}

Cell fate control represents only a subset of challenges associated with deploying ACT. Beyond manipulating biological networks, systems thinking can also be applied on a larger scale to improve the vein-to-vein supply network of ACT. The complexity of many artificial systems in today's hyperconnected world is increasingly approaching that of biological systems $[40,41]$. This highlights the difficulty in manufacturing and supplying ACT therapies where artificial and biological components intertwine. Current ACT protocols typically follow a centralised approach tailored for large-scale ex vivo bioprocessing at often distant sites, before reinfusion into hospitalised patients [42]. Accessibility and scalability are hampered by high costs, space requirements, error susceptibility, product variability and slow turnaround [43]. 
Current costs for FDA-approved CAR-T drugs include neither pre-treatment patient conditioning (e.g. lymphodepleting chemotherapy) nor post-infusion inpatient care (often involving side effect management in intensive care units). Uptake of these promising therapies are therefore at risk due to their high overall cost (https://reut.rs/2xmyFmt, https://bit.ly/2zL6Q8e).

The long processing time of T cell therapy and need for specialist infrastructure mean that patients with aggressive malignancies may not survive long enough, or cannot travel far enough, to receive treatment. Scaling up with automation may address these issues by delivering therapies to patients more quickly. Companies have developed automated cell bioprocessors - for example, the CliniMACS Prodigy (Miltenyi Biotech), WAVE Bioreactor (GE Life Systems) and G-Rex flask (Wilson Wolf Manufacturing) [44]. While most commercial systems have limited processes capabilities, the CliniMACS Prodigy can perform all major unit operations in one device - from selection, stimulation and gene delivery to expansion.

In addition to cost reduction, CliniMACS Prodigy was reported to reduce CAR-T production time from $\geq 13$ days to 8 days [45]. However, these approaches require significant capital expenditure for purchase of the device and ongoing maintenance [44]. Moreover, scaling up product production might be challenging, as many devices would be needed to produce multiple doses in parallel [46]. These factors mean that the goal of "decentralising" ACT manufacturing will be limited to well-financed institutions.

The use of implantable artificial cell niches for in vivo priming and activation of immune cells could circumvent limitations of ex vivo processing. A pioneering example is the scaffold-based cancer vaccine developed by the Mooney Lab [47]. The vaccine can be implanted to recruit dendritic cell (DC) populations of the host, via local release of granulocyte-macrophage colony stimulating factor (GM-CSF), and activation in situ with tumour antigens and cytosine-phosphate-guanine (CpG) embedded in a porous poly-lactide-co-glycolide (PLG) matrix. DCs stimulated by the vaccine can then migrate to lymph nodes where they activate $T$ cells to mount anti-tumour responses. Cancer vaccines can also be in injectable formats, such as 
polymeric nanoparticles, hydrogels and self-assembled mesoporous silica rods, among others [48]. In addition, in situ programming has recently been demonstrated on the CAR-T front, with the use of polymeric nanocarriers to deliver CAR genes into host circulating T cells [49]. A caveat to in situ immune manipulation using materials is the limited control following implantation. Molecular cargos (e.g. cytokines and antigens), along with other material properties, will need to be pre-adjusted to avoid over- or under-stimulating the immune system. Personalisation of such cancer vaccines might also be lengthy and costly.

\section{Bridging natural and human-engineered networks}

In contrast to the traditional, linear pharmaceutical manufacturing model, distributed manufacturing (Figure 3) could be adopted to improve the service of ACT in the age of Industry 4.0. Here, we describe how this new manufacturing concept could be technically implemented on various fronts.

Applying systems thinking, the vein-to-vein supply network can be rewired to push both production and customisation capabilities out to the end users (clinicians, in this context). This would reduce costs by removing multiple intermediaries (e.g. cryogenic shipment). While current point-of-care protocols focus on cells, advances in bioengineering have meant that personalisation for individual patients can be extended to immunomodulatory materials. A further reduction in ACT costs could come from the manufacture of custom materials at the point of care.

Complementing this approach is additive manufacturing, which could potentially be employed to 3D print immunomodulatory hydrogel scaffolds on demand [50]. Material properties, optimised using the aforementioned dynamical systems model for different phenotypes, may therefore be digitally shared among decentralised platforms worldwide. The enhanced flexibility conferred by this manufacturing model would allow clinicians to establish adaptive therapeutic strategies.

To further unify robustness with adaptiveness in delivering ACT, a potential approach to bioprocessing might involve direct interfacing between the patient and engineered hardware. While still nascent, this kind of idea has already been 
implemented in other areas of biomedicine - wearable electronic insulin pumps (e.g. Medtronic's MiniMed Paradigm ${ }^{\circledR}$ Veo $^{\mathrm{TM}}$ System) and automated wearable artificial kidneys (AWAK) [51]. In this sense, immunomodulatory hardware may be temporarily interfaced directly with the patient's immune system via the bloodstream but placed extracorporeally. The remote device would allow for automatic or manual override (e.g. detachment or flow rate adjustment), but remove the need for an intermediary apparatus. Systems based on a similar concept already exist (e.g. the indwelling intravascular aphaeretic circulating tumour cell isolation system developed by Kim and colleagues [52]). In practice, microfluidic technology may be employed for low-volume cell manipulation in a controlled microenvironment.

Combined with hydrogel technologies, it is plausible to imagine a fluidic system that can reduce unit operations with simultaneous capture and stimulation of $T$ cells using antibodies or antigens immobilised on hydrogels. Fluidic control could then handle specific timings for cell stimulation rounds and release. Here, the patient would act as the "bioreactor" for cell expansion downstream of the device. Of course, one must recognise the constraints of microfluidics, as current technologies may not be able to miniaturise or integrate all unit operations employed in advanced versions of immunotherapy, such as CAR-T.

Furthermore, it is also important to avoid the pitfall of automating every manual lab procedure (see Box 3 ). Therapies that might benefit most from the proposed technology would be those that do not require genetic modification, such as tumour-infiltrating lymphocytes (TILs) [53]. Building such immune systemmachine interfaces would give new meaning to adoptive immunotherapy where the cell-processing hardware is also adopted by the patient and establish a cyborg-like relationship between them. This concept is similar to that outlined by Pataranutaporn and colleagues in [54] where the wearable device no longer just performs sensing, but also provides real-time feedback to regulate the body.

To speed up bioprocessing, quality control and release procedures will need to concurrently evolve to ensure products meet clinical standards. Nevertheless, the production of therapeutic T cells still relies on costly, labour-intensive and time-consuming assays performed on end products. Taking inspiration from how 
biological systems self-regulate, it would be appealing to build control feedback loops into the processing system for auto-correction or auto-stabilisation. This goal is, however, complicated by the current lack of tools for real-time cell monitoring. Model predictive control is also not yet standard practice in mammalian cell bioprocesses [55]. Instead, the reference frame could move away from biomolecule-centric methods and holistically think about other parameters for rapid measurement, such as biophysical signatures. For example, impedance spectroscopy is utilised to distinguish between activated and non-activated T cells in a label-free manner inside microfluidic channels [56]. Alternating current (AC) impedance, which can be combined with light scatter analysis, has also been employed in a micro flow cytometer to classify platelets, erythrocytes, granulocytes, monocytes and lymphocytes in whole blood [57]. To further miniaturise the system, future monitoring components might be fully integrated into the device materials (e.g. nanowire electronic scaffolds [58]) that they also capture and stimulate $\mathrm{T}$ cells.

The use of control mechanisms is not limited to local control of cell products but can be exploited to regulate an entire network of bioprocessing systems at different treatment centres. For instance, machine learning algorithms can be trained to recognise different cell attributes from data collected by biosensors at different sites. The improved model can then be relayed back to all systems via a cloud-based service. Thus, output from local sensor components can lead to a network-wide enhancement of manufacturing robustness over time. The feasibility of cloud-based machine learning has already been demonstrated by services such as Google Cloud Al and IBM Watson (Figure 3).

Many of the concepts described thus far have the common goal of achieving "process intensification" - a concept that has already been embraced by other engineering disciplines for at least two decades [59]. It refers to the design and implementation of dramatically scaled-down approaches that lead to higher processing efficiencies. To this end, the proposed designs and strategies draw inspiration from biological systems in terms of robustness, flexibility and integration across scale. The reasoning behind the application of bioinspiration is 
precisely because biological systems have evolved over eons to be intrinsically intensified (Box 4). 


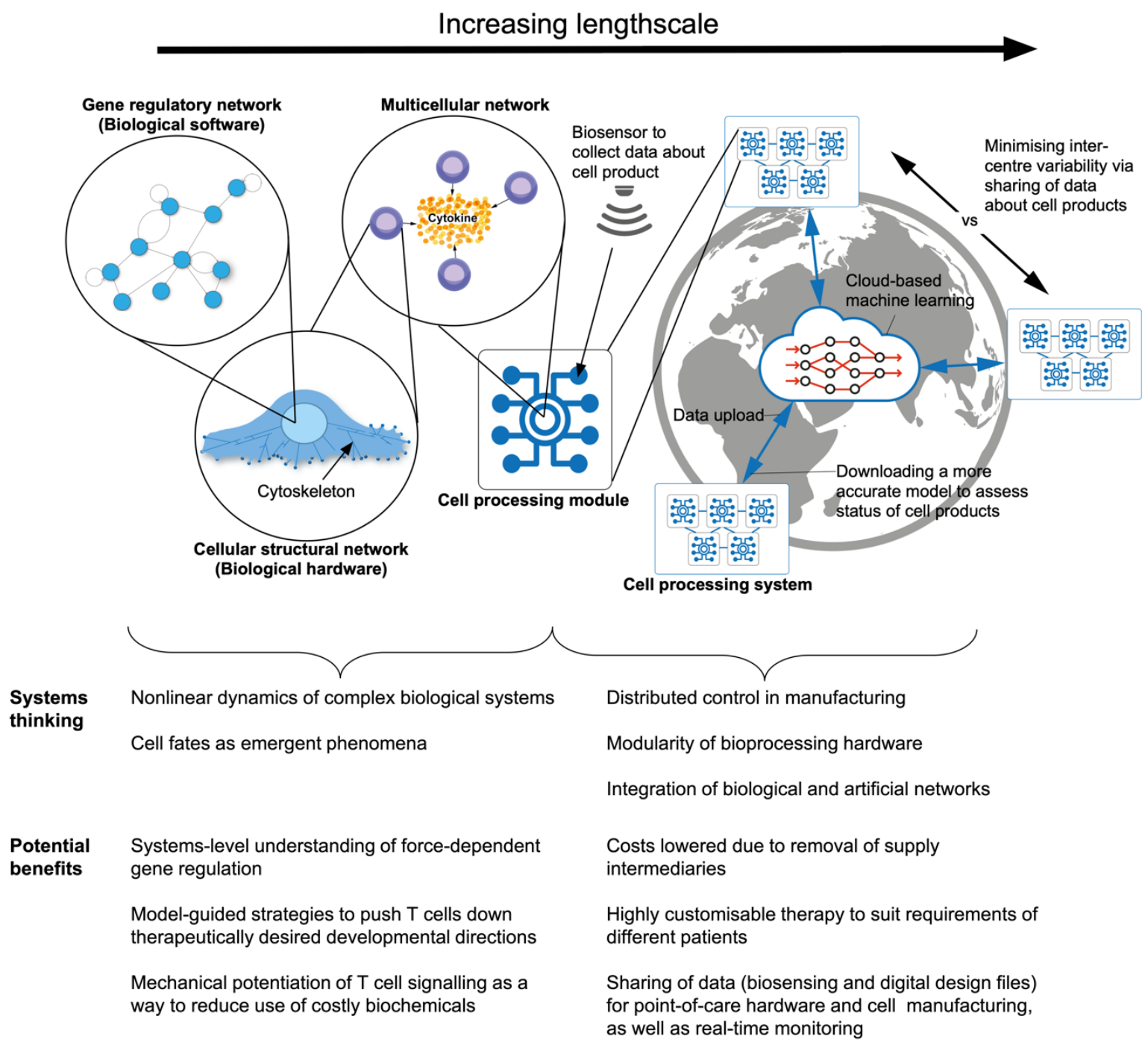

Figure 3 Adopting a multiscale, "networks-of-networks" paradigm to deploy cell-based cancer immunotherapies. Living and non-living (hardware/software) systems that are dealt with in cancer immunotherapy can themselves be regarded as parts of larger systems. Network biology-based computational frameworks may be employed to predict specific physiochemical cues needed to stimulate $T$ cells into the desired phenotype. The information can then be used to design artificial cell niches (e.g. hydrogel scaffolds) for the cell processing module (e.g. microfluidic device) within which $T$ cells are stimulated and activated. Driven by fluid flow, the cells could be allowed to interact with immunostimulatory materials and subsequently pass through the device. Extra material-cell separation steps would, therefore, be unnecessary. The cell processing module may be in the form of portable hardware or be docked with a larger automated system for downstream processing. In the former, one way to view this concept is that the extracorporeal hardware would simply be a "plug-in" to augment the existing immune system - just like mobile applications on smartphones. The latter example would be analogous to how smartphones are docked with speakers, where the phone is in charge of upstream fine-tuning and the speaker amplifies the output (cell expansion). In-process monitoring and control are performed throughout manufacturing. The data gathered from different systems or centres may be aggregated through cloud services to train computational models for more accurate identification of parameters, such as cell activation status, cell type etc. 


\section{Concluding remarks}

Both the natural and human-engineered worlds tend to form networks. These networks lead to complex systems, which exhibit emergent properties: they contain irreducible levels of organisation, unamenable to reductive analysis. In biology, networks manifest across multiple scales in the form of interacting molecules, cells, tissues and organs. Similarly, humans have self-assembled into, or created, physical and digital networks, such as social networks, logistic networks, power grids, the World Wide Web and Internet of Things (IOT). The rise of cell-based immunotherapies, such as CAR-Ts and TILs, have not only offered a new hope in treating previously untreatable cancers, but also exemplify a hybrid network where biotic and abiotic components directly and dynamically interact. The multiscale and interdependent challenges of this complex medical treatment, therefore, warrant systems thinking to complement reductionistic work, in order to improve scalability and controllability.

Knowing how to exploit complexity is just as important as recognising it in the massively modular, distributed systems that permeate cancer immunotherapy. Applying a systems lens, we may take inspiration from the relations among immune "modules" - lymphoid organs and T cells - to engineer new ways of bioprocessing. Encouraged by the development of AWAK devices [60] and AWAK Technologies [51], we believe there is potential to create automated, portable bioprocessing platforms that act as artificial extensions of the patient's immune system to fast-track therapeutic T cell delivery. On the cellular level, we foresee materials for $\mathrm{T}$ cell stimulation to exploit the coupling between gene regulatory and cytoskeletal networks by using stiffness, shape, topography and surface ligand spacing. Lessons may be drawn from how mechanical and biochemical factors synergise to potentiate $\mathrm{T}$ cell activation and steer differentiation [34].

Technical issues should be overcome to realise the concepts outlined in this Opinion, as complex system design has been relatively unexplored in cell therapies (see Outstanding Questions). Tackling them would require a multidisciplinary effort at almost every length and time scale of the vein-to-vein manufacturing journey of the "living drug". All in all, it requires us to combine 
holistic and atomistic perspectives - only then can we truly engineer immunotherapy at the "edge of chaos".

\section{Box 1. Cell fates emerge from complex dynamics}

From a complex systems perspective, cell phenotypes (or cell fates in general) can be considered as emergent phenomena arising from the numerous nonlinear interactions among genomic (e.g. GRNs), non-genomic (e.g. cytoskeletal and metabolic networks), as well as environmental components (e.g. extracellular matrix). Stuart Kauffman, Robert May and others have extensively explored the application of complexity science in biology [7,61-63]. To explain phenotype emergence, Kauffman borrowed a tool from the physical sciences known as "phase space", or "state space" [64]. Here, state space is a graphical representation of every theoretically possible state of the system. Changes in a system's behaviour over time, including possible phase transition, will, therefore, be equivalent to tracing trajectories from one point in state space to another.

In a biological context, stable cell fates (e.g. cell types) correspond to stable gene expression patterns and are represented in state space as "attractors" (stable steady states), where trajectories converge [65]. The attractor hypothesis suggests that when a cell is exposed to a large enough external perturbation, the cell will be tipped into a neighbouring attractor, corresponding to a particular cell fate. The number of attractors a cell can access at any point in time will depend on the trajectories available, which are, in turn, constrained by the wiring of the GRN.

Consolidating the complex systems perspective are cell differentiation experiments by Huang and colleagues, which revealed that cell fate decisions are collectively made by an entire network of genes and proteins $[12,66]$. Their work supports the notion that complex GRNs maintain cells in a self-stabilising attractor state yet enable them to switch between states (differentiate) under the right conditions. Interestingly, the view that cell types are attractors had been metaphorised by Waddington as valleys in his "epigenetic landscape" before molecular biologists started studying the topic through analysis of linear pathways and specific molecules.

\section{Box 2 Cellular mechanobiology}

Over the past few decades, various reports have described the crucial role of mechanics in many biological processes, such as cell fate commitment, migration and morphology [67]. Importantly, mechanical cues (e.g. substrate stiffness) from the extracellular matrix (ECM) can influence intracellular biochemistry via mechanotransduction. Adherent cells typically attach to the ECM via dynamic molecular assemblies known as focal adhesions (FAs). The extracellular 
portion of FAs comprises of specific cell surface proteins, integrins, that bind to ECM ligands, whereas the intracellular portion acts both as a linkage to the cytoskeleton and hub for multiple signalling pathways. Cell intrinsic forces generated by the actomyosin cytoskeleton are propagated to FAs and become traction forces, which are resisted by substrate stiffness. Stiffness is known to affect FA clustering, assembly and turnover, which in turn regulate downstream signals and mechanical feedback (e.g. via the Rho/ROCK pathway). Moreover, the mechanical coupling between the cytoskeleton and nucleus enables cytoskeletal forces to directly alter chromatin structures and influence gene transcription [68]. Mechano-regulation is therefore an interplay between the information-processing networks (e.g. GRNs) and structural networks (e.g. the cytoskeleton) in an open system (the cell) responsive to extrinsic physical cues. Indeed, the loop that connects microenvironmental mechanics and intracellular biochemistry has been widely acknowledged and reviewed $[69,70]$.

\section{Box 3 Learning from Theranos's mistakes}

Theranos was a health technology company that specialised in developing integrated blood testing devices with the aim of reducing the cost of blood test assays and overcoming geographic barriers to patient access. However, their devices, such as the "miniLab", were found to be highly unreliable. One of the major reasons was that Theranos tried to achieve automated, scaled-down laboratory tests with a conventional Cartesian pipette-wielding robot to mimic what the scientist does at the bench - this approach required frequent recalibrations due to pipette accuracy drifts. Furthermore, the miniLab consisted of an agglomeration of various standard technologies (including a spectrophotometer, fluorometer, cytometer, isothermal detector, centrifuge, camera, sonicator, among others) packed into one box (approximately 71 L). As a result, the miniLab's internal instruments interfered with each other, due to thermal issues and robotic misalignment. The miniLab has, therefore, demonstrated that miniaturising and automating every standard lab procedure is not an optimal approach for process intensification (https://www.wired.com/story/a-new-look-inside-theranos-dysfunctional-corporateculture/). If T cell processing is to be intensified, then there is a need to seek out ways to minimise unit operations and utilise technologies that are developed for low-volume environments, rather than the laboratory bench.

\section{Box 4 Human-engineered networks as extensions of biological networks}

A universal property that traverses cells, organs, organisms, social networks and even cities is that all of them essentially exist as systems within systems, or networks within networks. Indeed, it has even been argued that the whole universe may be considered as a single complex system, consisting of many complex sub-systems [71]. These far-from-equilibrium systems all require highly efficient ways to transfer energy, resources or information, as well as remove wastes, in order to function and maintain their organisation. In this coarse-grained 
sense, human-engineered constructs such as highways in cities are functionally akin to vascular systems in mammals and plants. Following Mandelbrot's discovery of ubiquitous fractal geometries in nature [72], described by scaling laws and characterised by fractal dimensions, there have been many studies on how human-engineered networks have similar scaling properties to biological ones. West and colleagues [73] have posited that biological systems, regardless of niche and evolutionary history, exploit fractal, space-filling geometry to maximise overall energy efficiency, irrespective of size. This exemplifies how biological systems are intrinsically "process-intensified". Nature-inspired chemical engineering (NICE) uses such principles for highly efficient, scalable fluid distribution and catalytic processes [74]. How this organismal perspective can be projected onto the practical design of cell processing technologies remains relatively unexplored. However, distilling the fundamental similarities among biological systems or networks might inspire the way scalable cell processing systems are developed - for example, by making use of process control loops and integration across scales.

\section{Outstanding Questions}

- What are the "nodes" in T cell regulatory networks that need to be perturbed (by biochemical or mechanical means, or a combination) in order to generate therapeutically desirable cell types such as T memory stem cells?

- What would be the input fluid and flow parameters needed for a scaled-down T cell processor to effectively stimulate $T$ cells?

- What type(s) of biosensing should be utilised to achieve, ideally, real-time and label-free monitoring?

- What kind of biophysical markers can we measure on a scaled-down platform in order to reliably classify, for example, $T$ cell types?

- How should data be processed and shared in a potentially massive, nonlinear, IoTbased supply network to reduce latency?

\section{Glossary}

Ancillary material: a material or reagent employed in manufacturing that has an effect on the cell therapy product, but not intended to be part of the final product.

Checkpoint inhibitors: Antibody-based therapeutics that act by blocking cell surface proteins, known as immune checkpoints (e.g. PD-L1), commonly utilised by tumours to deactivate T cells and achieve "immune escape". The blockade of checkpoints provides a means to restore immunogenicity. 
Co-evolution: two or more agents of a system change/adapt together over time due to their interactions with one another and with the environment.

Complex system: a system consisting of many parts that interact in a nonlinear fashion to create synergies, such that, at least, the system's outputs are not directly proportional to its inputs, and there is no obvious mapping between cause and effect.

Cyborg: a contraction of "cybernetic organism" coined by Clynes and Kline in "Cyborgs and Space" (Aeronautics, 1960). According to their definition, it refers to "the exogenously extended organisational complex functioning as an integrated homeostatic system unconsciously".

Emergence: a global or "macro-level" property exhibited by a complex system, but not by its individual, "micro-level" members.

Epigenetics: according to Waddington's original meaning, it refers to processes beyond genes that give rise to phenotypes. The modern usage, however, has a narrow and molecular meaning that refers to a set of DNA and histone modifications - this move was criticised as an "onomasiologically unfortunate choice" [23] by Huang, who has also provided an excellent explanation for why that is the case in [75].

Exhaustion (of T cells): a T cell's loss of effector function due to prolonged stimulation.

Far-from-equilibrium: a term in thermodynamics describing a system that is constantly changing within a transition space between order and chaos.

Holism: the philosophical idea that systems should be viewed as a whole, rather than a collection of parts. The meaning of each part cannot be considered without reference to the whole.

Industry 4.0: A term associated with the $4^{\text {th }}$ industrial revolution, characterised by decentralised ecosystems of manufacturing, as well as the use of "cyber-physical systems" where physical and computer capabilities are integrated (e.g. loT, sensors and machine learning). Enabled by digitalisation, products and services in this paradigm are typically developed rapidly and in small lots to meet the diverse and changing demands of end users. Products also become highly modularised and customisable to allow for "reverse logistics", where platforms can be repurposed via upgrades or replacement of modules.

Lymphodepletion: the destruction of normal lymphocytes, usually by radiation or chemotherapy, prior to T cell therapy. This treatment is administered for the purpose of skewing the T cell repertoire towards tumour-reactive T cells to improve effectiveness of the therapy.

Model Predictive Control: a process control method that aims to optimise control parameters based on dynamical models that make predictions about the system's future behaviour.

Nonlinearity: the output of the system is not proportional to the input; the whole is more than the sum of its parts.

Reductionism: the philosophical idea that any phenomenon can be readily inferred from more fundamental, simpler processes.

System: a collection of parts that, through relations between them, function together to generate a common response.

T cell stimulation: the process of delivering signals to the $T$ cell receptor and costimulatory receptors (e.g. CD28) to elicit T cell activation. These stimulatory signals, as well as various environmental inputs, are integrated by the T cell signalling networks and converted into functional outputs, such as cytokine secretion, differentiation and clonal expansion.

Transdifferentiation: conversion of a mature cell type into another mature cell type without going through an intermediate, pluripotent cell state.

\section{Acknowledgments}


This work was supported by the Biotechnology and Biological Sciences Research Council (BBSRC) (grant number BB/M009513/1). M.C., M.O.C. and R.D. also gratefully acknowledge support from the EPSRC via 'Frontier Engineering' and 'Frontier Engineering: Progression' awards (grant numbers EP/K038656/1 and EP/S03305X/1).

\section{References}

1 Ribas, A. and Wolchok, J.D. (2018) Cancer immunotherapy using checkpoint blockade. Science 359, 1350-1355

2 Guedan, S. et al. (2019) Emerging Cellular Therapies for Cancer. Annu. Rev. Immunol. 37, 145-171

3 Maude, S.L. et al. (2018) Tisagenlecleucel in Children and Young Adults with B-Cell Lymphoblastic Leukemia. N. Engl. J. Med. 378, 439-448

4 Goldenfeld, N. and Kadanoff, L.P. (1999) Simple lessons from complexity. Science 284, 87-89

5 Carron, P. Mac and Kenna, R. (2012) Universal properties of mythological networks. EPL 99, 28002

6 Battiston, S. et al. (2016) Complexity theory and financial regulation. Science $351,818-819$

7 Kauffman, S.A. (1993) The Origins of Order: Self-Organization and Selection in Evolution, Oxford University Press.

8 Waldrop, M.M. (1992) Complexity: The Emerging Science at the Edge of Order and Chaos, Simon \& Schuster.

9 Ahn, A.C. et al. (2006) The Limits of Reductionism in Medicine: Could Systems 
Biology Offer an Alternative? PLoS Med. 3, e208

10 Van Regenmortel, M.H. V and Van Regenmortel, M.H. V (2019) The Rational Design of Biological Complexity: A Deceptive Metaphor. In HIV/AIDS: Immunochemistry, Reductionism and Vaccine Design pp. 87-102, Springer International Publishing

11 Huang, S. and Kauffman, S.A. (2012) Complex Gene Regulatory Networks from Structure to Biological Observables: Cell Fate Determination Gene regulation, cell fate determination. In Computational Complexity pp. 527-560, Springer New York

12 Huang, S. et al. (2005) Cell Fates as High-Dimensional Attractor States of a Complex Gene Regulatory Network. Phys. Rev. Lett. 94, 128701

13 Maeda, H. and Khatami, M. (2018) Analyses of repeated failures in cancer therapy for solid tumors: poor tumor-selective drug delivery, low therapeutic efficacy and unsustainable costs. Clin. Transl. Med. 7, 11

14 Brock, A. and Huang, S. (2017) Precision Oncology: Between Vaguely Right and Precisely Wrong. Cancer Res. 77, 6473-6479

15 Besse, B. et al. (2018) Hyperprogressive Disease in Patients with Advanced Non-Small Cell Lung Cancer Treated with PD-1/PD-L1 Inhibitors or with Single-Agent Chemotherapy. JAMA Oncol. 4, 1543-1552

16 Rosenberg, J. and Huang, J. (2018) CD8+ T cells and NK cells: parallel and complementary soldiers of immunotherapy. Curr. Opin. Chem. Eng. 19, 9-20

17 McLellan, A.D. and Ali Hosseini Rad, S.M. (2019) Chimeric antigen receptor T cell persistence and memory cell formation. Immunol. Cell Biol. 97, 664-674

18 Wang, X. et al. (2016) Phase 1 studies of central memory-derived CD19 CAR 
T-cell therapy following autologous HSCT in patients with B-cell NHL. Blood $127,2980-2990$

19 Blaeschke, F. et al. (2018) Induction of a central memory and stem cell memory phenotype in functionally active CD4+ and CD8+ CAR T cells produced in an automated good manufacturing practice system for the treatment of CD19+ acute lymphoblastic leukemia. Cancer Immunol. Immunother. 67, 1053-1066

20 Chang, Z.L. et al. (2015) Identification and selective expansion of functionally superior T cells expressing chimeric antigen receptors. J. Transl. Med. 13, 161

21 Chakraborty, A.K. and Das, J. (2010) Pairing computation with experimentation: a powerful coupling for understanding $T$ cell signalling. Nat. Rev. Immunol. $201010110,59-71$

22 Waddington, C.H. (1935) How Animals Develop, (1st edn) George Allen \& Unwin, Ltd.

23 Huang, S. Reprogramming cell fates: reconciling rarity with robustness. BioEssays 31, 546-560

24 Huang, S. and Kauffman, S. (2013) How to escape the cancer attractor: Rationale and limitations of multi-target drugs. Semin. Cancer Biol. 23, 270278

25 Bargaje, R. et al. (2017) Cell population structure prior to bifurcation predicts efficiency of directed differentiation in human induced pluripotent cells. Proc. Natl. Acad. Sci. U. S. A. 114, 2271-2276

26 Wang, J. et al. (2011) Quantifying the Waddington landscape and biological paths for development and differentiation. Proc. Natl. Acad. Sci. U. S. A. 108, 8257-8262 
27 Furusawa, C. and Kaneko, K. (2012) A dynamical-systems view of stem cell biology. Science 338, 215-217

28 Wong, S.Y. et al. (2017) Biophysical regulation of cell reprogramming. Curr. Opin. Chem. Eng. 15, 95-101

$29 \mathrm{Li}, \mathrm{Y}$. and Kurlander, R.J. (2010) Comparison of anti-CD3 and anti-CD28coated beads with soluble anti-CD3 for expanding human T cells: Differing impact on CD8 $\mathrm{T}$ cell phenotype and responsiveness to restimulation. $J$. Transl. Med. 8, 104

30 Snook, J.P. et al. (2018) TCR signal strength controls the differentiation of CD4+ effector and memory T cells. Sci. Immunol. 3, eaas9103

31 Buck, M.D. et al. (2016) Mitochondrial Dynamics Controls T Cell Fate through Metabolic Programming. Cell 166, 63-76

32 Kim, S.T. et al. (2009) The alphabeta T cell receptor is an anisotropic mechanosensor. J Biol Chem 284, 31028-31037

33 Judokusumo, E. et al. (2012) Mechanosensing in T lymphocyte activation. Biophys J 102, L5-7

34 Hickey, J.W. et al. (2019) Engineering an Artificial T- Cell Stimulating Matrix for Immunotherapy. Adv. Mater. 31, 1807359

35 Harrison, D.L. et al. (2019) T-Cell Mechanobiology: Force Sensation, Potentiation, and Translation. Front. Phys. 7, 45

36 González-Granado, J.M. et al. (2014) Nuclear envelope lamin-A couples actin dynamics with immunological synapse architecture and T cell activation. Sci. Signal. 7, ra37-ra37 
37 Lim, W.A. and June, C.H. (2017) The Principles of Engineering Immune Cells to Treat Cancer. Cell 168, 724-740

38 Dang, A.P. et al. (2018) Enhanced Activation and Expansion of T Cells Using Mechanically Soft Elastomer Fibers. Adv. Biosyst. 2, 1700167

39 Peng, T. et al. (2017) A mathematical model of mechanotransduction reveals how mechanical memory regulates mesenchymal stem cell fate decisions. BMC Syst. Biol. 11, 55

40 Csete, M.E. and Doyle, J.C. (2002) Reverse engineering of biological complexity. Science 295, 1664-1669

41 Solé, R. V. et al. (2011) Convergent Evolutionary Paths in Biological and Technological Networks. Evol. Educ. Outreach 4, 415-426

42 Eyles, J.E. et al. (2019) Cell therapy products: focus on issues with manufacturing and quality control of chimeric antigen receptor T-cell therapies. J. Chem. Technol. Biotechnol. 94, 1008-1016

43 lyer, R.K. et al. (2018) Industrializing Autologous Adoptive Immunotherapies: Manufacturing Advances and Challenges. Front. Med. 5, 150

44 Fesnak, A.D. et al. (2017) Considerations in T Cell Therapy Product Development for B Cell Leukemia and Lymphoma Immunotherapy. Curr. Hematol. Malig. Rep. 12, 335-343

45 Zhang, W. et al. (2018) Characterization of clinical grade CD19 chimeric antigen receptor T cells produced using automated CliniMACS Prodigy system. Drug Des. Devel. Ther. 12, 3343-3356

46 Dwarshuis, N.J. et al. (2017) Cells as advanced therapeutics: State-of-the-art, challenges, and opportunities in large scale biomanufacturing of high-quality 
cells for adoptive immunotherapies. Adv. Drug Deliv. Rev. 114, 222-239

47 Ali, O.A. et al. (2009) In situ regulation of DC subsets and T cells mediates tumor regression in mice. Sci Transl Med 1, 8ra19

48 Zhang, R. et al. (2018) Biomaterials for vaccine-based cancer immunotherapy. J. Control. Release 292, 256-276

49 Smith, T.T. et al. (2017) In situ programming of leukaemia-specific T cells using synthetic DNA nanocarriers. Nat. Nanotechnol. 12, 813-822

$50 \mathrm{Li}$, J. et al. (2020) 3D printing of hydrogels: Rational design strategies and emerging biomedical applications. Mater. Sci. Eng. R Reports 140, 100543

51 Htay, H. et al. (2019) Evaluation of Safety of Automated Wearable Aetificial Kidney (AWAK) Device in Peritoneal Dialysis Patients. Kidney Int. Reports 4, S183

52 Kim, T.H. et al. (2019) A temporary indwelling intravascular aphaeretic system for in vivo enrichment of circulating tumor cells. Nat. Commun. 10, 1478

53 Tavera, R.J. et al. (2018) Utilizing T-cell Activation Signals 1, 2, and 3 for Tumor-infiltrating Lymphocytes (TIL) Expansion: The Advantage over the Sole Use of Interleukin-2 in Cutaneous and Uveal Melanoma. J. Immunother. 41, 399-405

54 Pataranutaporn, P. et al. (2019) Wearable Lab on Body: Combining Sensing of Biochemical and Digital Markers in a Wearable Device. Proc. Annu. Int. Conf. IEEE Eng. Med. Biol. Soc. 2019, 3327-3332

55 Sommeregger, W. et al. (2017) Quality by control: Towards model predictive control of mammalian cell culture bioprocesses. Biotechnol. J. 12, 1600546 
56 Rollo, E. et al. (2017) Label-free identification of activated T lymphocytes through tridimensional microsensors on chip. Biosens. Bioelectron. 94, 193199

57 Simon, P. et al. (2016) Label-free whole blood cell differentiation based on multiple frequency $\mathrm{AC}$ impedance and light scattering analysis in a micro flow cytometer. Lab Chip 16, 2326-2338

58 Tian, B. et al. (2012) Macroporous nanowire nanoelectronic scaffolds for synthetic tissues. Nat. Mater. 11, 986-994

59 Stankiewicz, A.I. and Moulijn, J.A. (2000) Process intensification: Transforming chemical engineering. Chem. Eng. Prog. 96, 22-34

60 Gura, V. et al. (2016) A wearable artificial kidney for patients with end-stage renal disease. JCI Insight 1, e86397

61 Nowak, M. and May, R.M. (2000) Virus Dynamics: Mathematical Principles of Immunology and Virology, (1st edn) Oxford University Press.

62 May, R.M. (2004) Uses and Abuses of Mathematics in Biology. Science 303, 790-793

63 Dinicola, S. et al. (2011) A systems biology approach to cancer: fractals, attractors, and nonlinear dynamics. OMICS 15, 93-104

64 Kauffman, S. (2004) A proposal for using the ensemble approach to understand genetic regulatory networks. J. Theor. Biol. 230, 581-590

65 Huang, S. et al. (2009) Cancer attractors: A systems view of tumors from a gene network dynamics and developmental perspective. Semin. Cell Dev. Biol. 20, 869-876 
66 Chang, H.H. et al. (2008) Transcriptome-wide noise controls lineage choice in mammalian progenitor cells. Nature 453, 544-7

67 Vining, K.H. and Mooney, D.J. (2017) Mechanical forces direct stem cell behaviour in development and regeneration. Nat. Rev. Mol. Cell Biol. 18, 728742

68 Uhler, C. and Shivashankar, G. V. (2017) Regulation of genome organization and gene expression by nuclear mechanotransduction. Nat. Rev. Mol. Cell Biol. 18, 717-727

69 Huang, S. et al. (2006) Tensegrity, Dynamic Networks, and Complex Systems Biology: Emergence in Structural and Information Networks Within Living Cells. In Complex Systems Science in Biomedicine (Deisboeck, T. S. and Kresh, J. Y., eds), pp. 283-310, Springer US

70 Gilmour, D. et al. From morphogen to morphogenesis and back., Nature, 541. 18-Jan-(2017) , Nature Publishing Group, 311-320

71 Chela-Flores, J. (2013) From systems chemistry to systems astrobiology: life in the universe as an emergent phenomenon. Int. J. Astrobiol. 12, 8-16

72 Mandelbrot, B.B. (1982) The Fractal Geometry of Nature, W.H. Freeman and Company.

73 West, G.B. et al. (1997) A general model for the origin of allometric scaling laws in biology. Science 276, 122-6

74 Coppens, M.-O. (2012) A nature-inspired approach to reactor and catalysis engineering. Curr. Opin. Chem. Eng. 1, 281-289

75 Huang, S. (2012) Tumor progression: Chance and necessity in Darwinian and Lamarckian somatic (mutationless) evolution. Prog. Biophys. Mol. Biol. 110, 
69-86

Page 30 of 30 